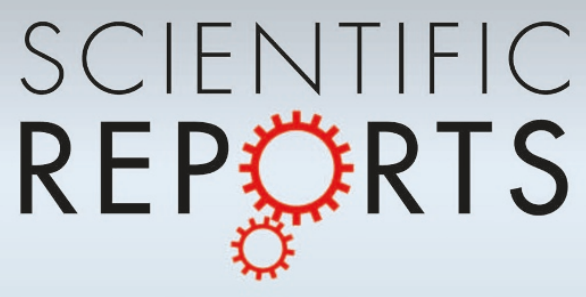

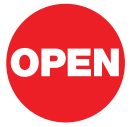

SUBJECT AREAS:

VIRUS-HOST

INTERACTIONS

DENGUE VIRUS

MECHANISMS OF DISEASE

COAT COMPLEXES

Received

16 January 2013

Accepted

22 April 2013

Published

9 May 2013

Correspondence and requests for materials should be addressed to G.R.M. Igmedigeshi@ thsti.res.in)

\section{Adaptor protein complexes- 1 and 3 are involved at distinct stages of flavivirus life-cycle}

\author{
Tanvi Agrawal' ${ }^{1}$ Peter Schu² \& Guruprasad R. Medigeshi'
}

'Vaccine and Infectious Disease Research Center, Translational Health Science and Technology Institute, Gurgaon- 122016. INDIA,
${ }^{2}$ Abteilung Biochemie II, Humboldtallee 23, Georg-August-Universität Göttingen, D-37073. Göttingen, GERMANY.

Intracellular protein trafficking pathways are hijacked by viruses at various stages of viral life-cycle. Heterotetrameric adaptor protein complexes (APs) mediate vesicular trafficking at distinct intracellular sites and are essential for maintaining the organellar homeostasis. In the present study, we studied the effect of AP-1 and AP-3 deficiency on flavivirus infection in cells functionally lacking these proteins. We show that AP-1 and AP-3 participate in flavivirus life-cycle at distinct stages. AP-3-deficient cells showed delay in initiation of Japanese encephalitis virus and dengue virus RNA replication, which resulted in reduction of infectious virus production. AP-3 was found to colocalize with RNA replication compartments in infected wild-type cells. AP-1 deficiency affected later stages of dengue virus infection where increased intracellular accumulation of infectious virus was observed. Therefore, our results propose a novel role for AP-1 and AP-3 at distinct stages of infection of some of the RNA viruses.

M embers of the genus Flavivirus within the family Flaviviridae comprise of several medically important pathogens including the Japanese encephalitis virus (JEV), dengue virus (DENV), yellow fever virus (YFV) and West Nile virus (WNV) which cause significant morbidity and mortality in humans, animals and birds ${ }^{1}$. Flaviviruses are enveloped viruses with a positive-sense, single-strand RNA genome of approximately $11 \mathrm{~kb}$ that is translated as a single polyprotein precursor of $\sim 3300$ amino acids in length and proteolytically cleaved into 10 viral proteins: three structural (capsid, pre-membrane/membrane (prM-M), and envelope) and seven non-structural (NS1, NS2A, NS2B, NS3, NS4A, NS4B, and NS5) proteins'. Flaviviruses enter their host cells through a process of receptor-mediated endocytosis followed by subsequent fusion within the endosomal compartment to release the viral genome into the cytoplasm for translation and replication ${ }^{2}$. Replication of the viral RNA genome occurs on virus-induced host cell membranes. Such structures may serve as a scaffold for anchoring the viral replication complexes, which consist of viral RNA, viral proteins, and host cell factors. Virus assembly occurs within the endoplasmic reticulum-derived membrane compartments and non-infectious virions traverse through the Golgi stack to reach the trans-Golgi network (TGN) where furin-mediated cleavage between prM-M leads to conformational changes rendering the virion infectious. Infectious virus is subsequently released from cells via the secretory pathway ${ }^{1,3}$.

Adaptor complexes (AP-1 through 5) are heterotetrameric protein complexes comprising one each of the two large sub-units $\gamma, \alpha, \delta, \varepsilon$ and $\zeta$ and $\beta 1-5$ and one medium sub-unit $\mu 1-5$ and one small sub-unit $\sigma 1-5$ respectively. APs are involved in distinct intracellular vesicular transport pathways which play a vital role in maintaining cellular homeostasis ${ }^{4-6}$. AP-1 is involved in the trafficking of cargo molecules in the biosynthetic pathway from the trans-Golgi network (TGN) to endosomes and back. AP-2 which is one of the most extensively-studied adaptor complex has been shown to be involved in the endocytic pathway at the plasma membrane. AP-3 is reported to function in the transport of selected proteins in the endo-lysosomal pathway. AP-4 is involved in sorting of proteins destined to basolateral surface in polarized cells. AP-5 is the newest member of the family discovered recently and has been proposed to function at the late endosomes ${ }^{6}$. Despite the prominent role played by APs in intracellular trafficking pathways, their involvement in flavivirus life-cycle has not been characterized. A number of earlier studies investigating the internalization of flaviviruses have shown the involvement of clathrin-dependent and lipid raft-dependent pathways for virus entry but the role of APs in stages post-entry has not been investigated ${ }^{2}$. A genome-wide RNA interference screen for identifying cellular proteins associated with WNV infection identified AP-1 $\mu 1$ subunit and AP-3 $\sigma 2$ subunit as some of the host factors required for both WNV and DENV infection ${ }^{7}$. Similarly, another study identified AP1M1 as one of the genes required for 
secretion of recombinant subviral particles of dengue virus 1 by using a siRNA screen of 122 genes involved in membrane trafficking ${ }^{8}$. However the exact role of AP-1 and AP-3 in flavivirus life cycle is poorly understood and it is not clear if AP-1 and AP-3 play redundant functional roles or are involved in distinct pathways crucial for virus infection. In this study, we have utilized cells lacking functional AP-1 or AP-3 and tested for the effect of this deficiency in DENV, JEV and WNV replication. We show that AP-3 plays an important role at earlier stages of viral life cycle whereas AP-1 is required at later stages suggesting that the flaviviruses differentially utilize the cellular trafficking pathways involving AP-1 and 3 at post-entry stages.

\section{Results}

AP-1-deficiency affects later stages of DENV and WNV infection. In order to understand the role of AP-1 in mosquito-borne flavivirus

a

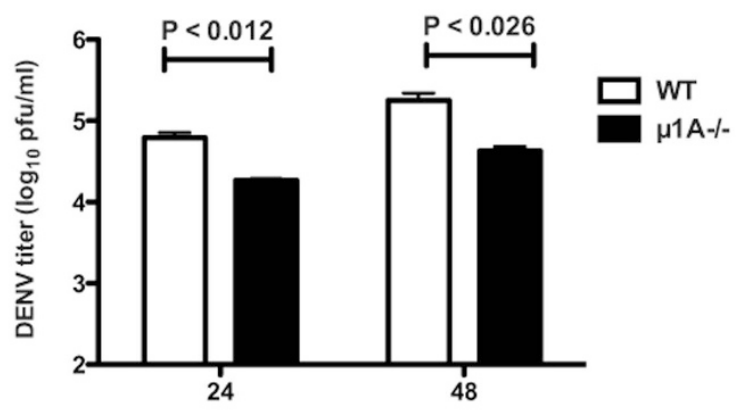

b

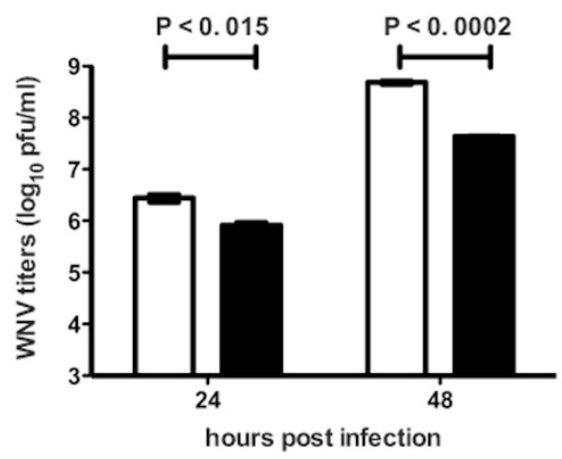

C

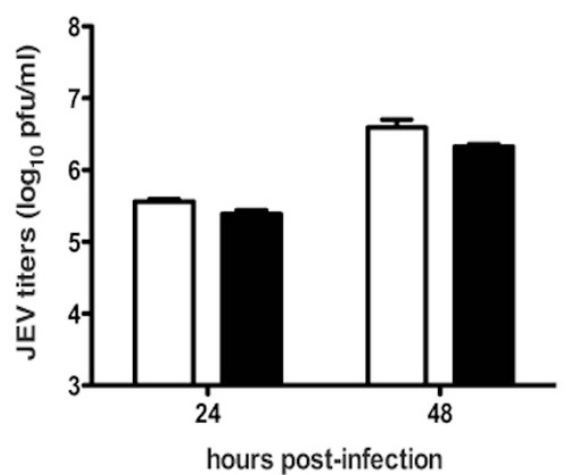

Figure $1 \mid$ Flavivirus production in AP-1-deficient cells. Wild type (WT) and AP-1-deficient ( $\mu 1 \mathrm{~A}-/-)$ MEFs were infected with 1 MOI of DENV-2 (a) or $0.3 \mathrm{MOI}$ of WNV (b) or JEV (c) and viral titers in culture supernatants were determined at indicated time points by plaque assay. Error bars represent Mean \pm SEM. Data are representative of three experiments performed with three replicates each. $P$ values were calculated by non-parametric, two-tailed, t-test. The difference was insignificant in JEV infected cells. life-cycle, we infected MEFs derived from wild-type and $\mu 1 \mathrm{~A}$ deficient (AP-1-deficient) mice with DENV or WNV or JEV and viral titers in the supernatants were measured at the indicated time points post-infection (pi) by plaque assays. Both DENV and WNV titers were significantly less at $24 \mathrm{~h}$ pi and the effect was much more prominent at $48 \mathrm{~h}$ pi possibly suggesting that AP-1 deficiency affects later stages of virus infection. Surprisingly, JEV titers were unaffected at both 24 and $48 \mathrm{~h}$ pi (Fig. $1 \mathrm{a}-\mathrm{c}$ ). AP-1 is localized at the TGN and endosomes and mediates protein sorting at these compartments 9 . Lack of AP-1 could specifically affect post-maturation events in DENV and WNV life-cycle and may lead to aberrant secretion of infectious virus. To test this, we infected wild type and AP-1-deficient MEFs with DENV and measured intracellular virus titers at 24 and $48 \mathrm{~h}$ pi. As expected, we observed enhanced intracellular viral titers in AP-1-deficient cells (Fig. 2a) at $48 \mathrm{~h}$ pi. This was also confirmed by qRT-PCR for dengue viral RNA levels in these cells which showed a significant increase in the amount of viral RNA at 48 hpi (Fig. 2b). These data confirm accumulation of infectious virions in these cells suggesting a defect in viral egress. We next tested if DENV infection affects the localization of AP-1 in Huh-7 cells. We observed partial colocalization of DENV with AP-1 and also with the TGN-resident protein TGN 46 in the perinuclear region at $24 \mathrm{~h}$ pi and DENV infection did not affect the localization of AP-1 as confirmed by unaltered colocalization of AP-1 with TGN46 (Fig. 3 and Figure S2a, S2b and S2c). Further, no colocalization was

a

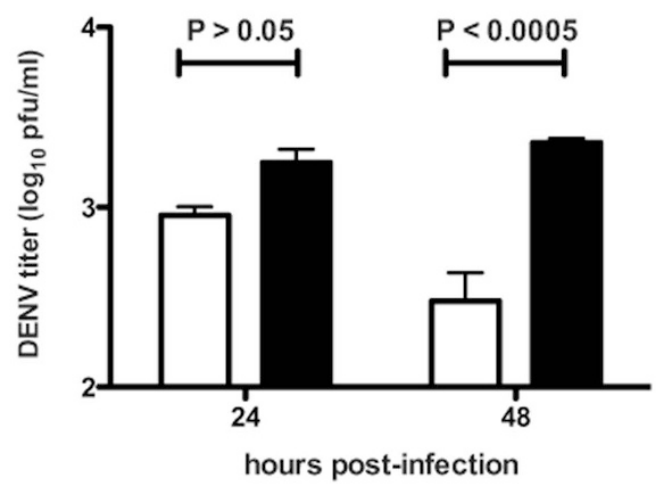

b

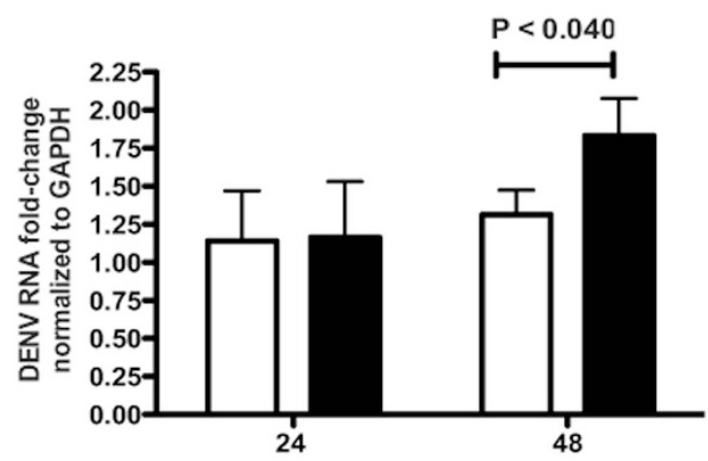

Figure $2 \mid$ AP-1 is required at later stages of DENV life-cycle. (a) MEFs were infected with DENV (MOI of $1 \mathrm{pfu} / \mathrm{cell}$ ) and at indicated time points cells were collected and intracellular viral titers were determined by plaque assay as described above. (b) Cells were infected with DENV (1 MOI) and at indicated time points infected cells were harvested for RNA isolation and dengue genome copy number determination by qRT-PCR. Error bars represent Mean \pm SEM. Data are representative of two experiments performed with three replicates. $\mathrm{P}$ values were calculated by non-parametric, two-tailed, t-test. 


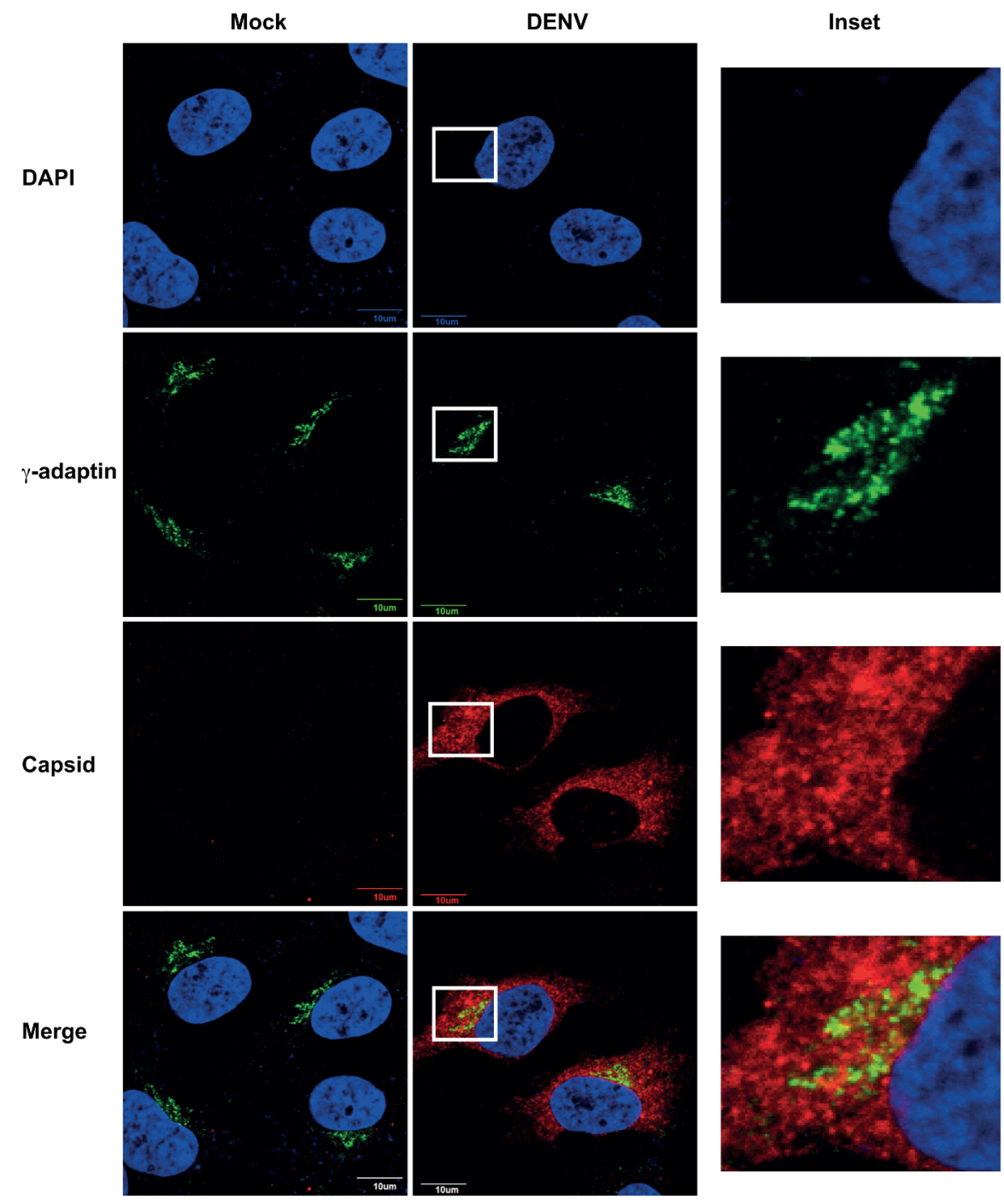

Figure $3 \mid$ DENV partially colocalizes with AP-1. Huh-7 cells were infected with DENV (3 MOI) and 24 h pi, cells were fixed and stained with antibodies against $\gamma$-adaptin and DENV-capsid followed by secondary antibodies conjugated with Alexa 488 and Alexa 568 respectively. Nuclei was stained with DAPI. Overlap image with a small region of interest showing colocalization is shown in the inset.

observed with the late endosome/lysosome marker LAMP-1 whereas we observed a positive correlation in the colocalization of capsid and AP-1 at the TGN (Figure S2b and S2c). These observations suggests that AP-1-mediated trafficking may be involved in the egress of some of the flaviviruses.

Effect of AP-3-deficiency on flavivirus life-cycle. Both AP-1 and AP-3 are involved in protein sorting at the TGN and endosomes. To further understand if AP- 1 and AP-3 play distinct or redundant roles in flavivirus infection, we infected MEFs derived from wild-type and AP-3-deficient (mocha) mice with DENV or WNV or JEV and viral titers in the supernatants were measured at the indicated time points by plaque assays. In AP-3-deficient MEFs, both JEV and DENV growth was severely impaired and the viral titers were 100-1000fold lower compared to wild-type MEFs at $48 \mathrm{~h}$ pi (Fig. 4 a and c). In case of $\mathrm{WNV}$, replication continued to progress even at $48 \mathrm{hpi}$, albeit at close to 100-fold lower levels in AP-3-deficient cells compared to wild-type cells (Fig. $4 \mathrm{~b}$ ). These results indicate that flaviviruses have differential requirement for AP-1 and AP-3. While AP-1 most likely participates at later stages of infection of DENV and WNV, AP-3 seems to play a major role in the replication of all the three flaviviruses tested. To assess if lack of AP-3 results in blockade of viral assembly and maturation or earlier stages of infection, we estimated the amount of intracellular virus titers upon JEV infection and observed a hundred-fold reduction in viral titers in AP-3-deficient cells (data not shown). We next prepared cell lysates from JEV-infected cells and performed western blot analysis for JEV-capsid protein. We found that while JEV-C levels in wild-type cells could be detected at $24 \mathrm{~h}$ pi which rapidly increased at $48 \mathrm{~h} \mathrm{pi}$, the same was below the level of detection in AP-3-deficient cells even at $48 \mathrm{~h}$ pi indicating a block prior to viral poly-protein translation (Fig. 4d). 
a

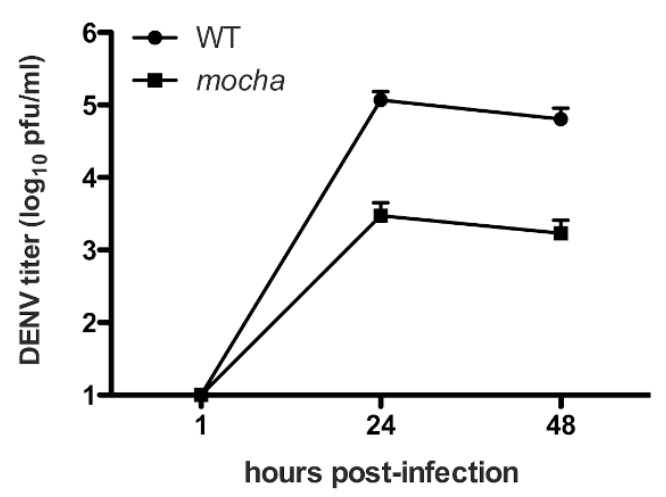

C

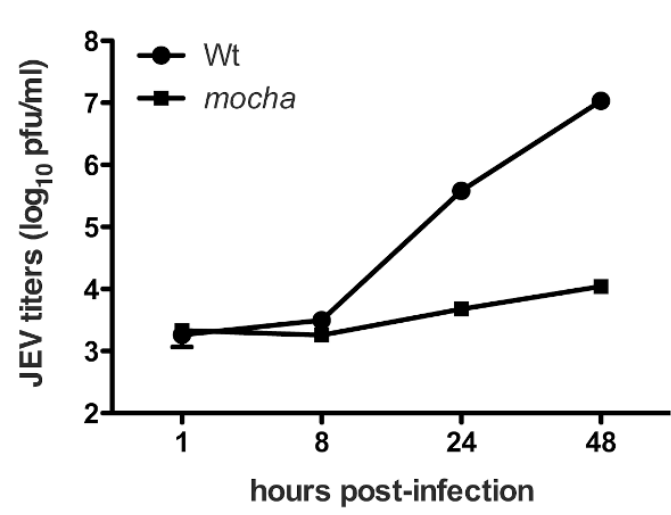

b

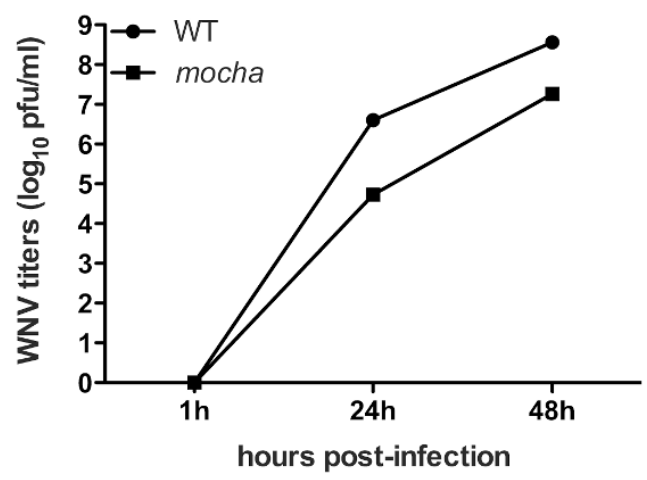

d

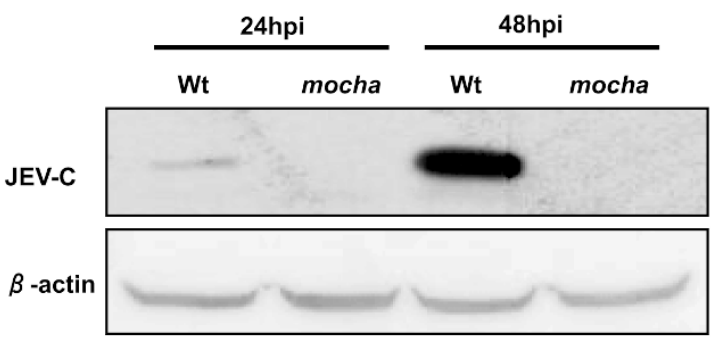

Figure 4 Effect of AP-3 deficiency on flavivirus infection. Wild type (WT) and AP-3-deficient ( mocha) MEFs were infected with an MOI of 1 pfu/cell of DENV-2 (a) or $0.1 \mathrm{pfu} / \mathrm{cell}$ of WNV (b) or $0.3 \mathrm{pfu} / \mathrm{cell}$ of JEV (c) and viral titers in culture supernatants were determined at indicated time points by plaque assay. Error bars represent Mean \pm SEM. Data are representative of three experiments performed with two or more replicates. (d) MEFs were infected with JEV and at indicated time points post-infection cell lysates were prepared and subjected to western blots and probed with JEV-capsid and $\beta$ actin antibodies.

Our results suggest that the lack of AP-3 affects JEV life-cycle at a stage prior to viral protein translation presumably at the level of viral entry, capsid disassembly or viral RNA replication. We next quantitated JEV and DENV genome copy numbers from infected wild-type and AP-3-deficient cells by qRT-PCR. Viral RNA levels were similar at $1 \mathrm{~h} \mathrm{pi}$, which indicates RNA from input virus, suggesting that there was no defect in viral entry. JEV RNA levels started to increase between 1 to $8 \mathrm{~h}$ pi and viral replication continued to increase till $48 \mathrm{~h}$ pi in wild-type cells. In contrast, in cells lacking AP-3, viral RNA replication did not begin until about $8 \mathrm{~h}$ pi and even at $48 \mathrm{~h}$ pi JEV RNA levels remained 100-fold lower in these cells compared to wildtype cells (Fig. 5a). The effect seemed much more drastic in DENV infection as viral replication remained at background levels even at $48 \mathrm{~h}$ pi (Fig. 5a). We performed indirect immunofluorescence analysis in wild-type and AP-3-deficient cells infected with JEV to visualize dsRNA to monitor the kinetics of viral RNA replication. We observed foci of dsRNA in wild-type cells as early as $4 \mathrm{~h}$ pi which increased by $8 \mathrm{~h}$ pi suggesting active replication of viral RNA. In AP3-deficient cells very few punctate dsRNA structures were visible even at $8 \mathrm{~h}$ pi further demonstrating a delay in initiation of RNA replication in these cells (Fig. 5b). These results indicate that AP-3 is involved at earlier stages of JEV and DENV life-cycle possibly at the level of viral RNA replication. Flavivirus RNA replication is reported to be semi-conservative wherein plus-strand RNA accumulates in excess in comparison to the minus-strand. Therefore, we investigated if AP-3-deficient cells have altered positive to negative strand ratio by performing strand-specific cDNA synthesis and quantitation of positive and negative strand RNA amounts by real-time PCR. Both wildtype and AP-3-deficient cells infected with JEV had approximately 20:1 ratio of positive to negative strand suggesting that the RNA synthesis per se is not affected (Fig. 6a). This indicates that the observed defect is due to non-availability of factors required for viral RNA replication or due to a delay/defects in steps prior to RNA replication namely viral-endosomal membrane fusion or capsid disassembly. To assess if the reduced amount of viral genome observed in AP-3-deficient cells was due to defective viral entry, we transfected wild type and AP-3-deficient cells with total RNA isolated from JEVinfected cells. This would circumvent the receptor-mediated entry event and enable direct initiation of viral RNA replication in the cytosol. We isolated RNA from transfected cells for estimation of viral genome copy numbers at $48 \mathrm{~h}$ post-transfection and also estimated viral titers from the supernatants. We observed a $90 \%$ reduction in JEV genome equivalents and undetectable virus in the culture supernatants in AP-3-deficient cells as compared to wild type cells (Fig. 6b \& 6c). Similar results were obtained from RNA isolated from purified culture supernatants from infected cells (data not shown). Quantitation of input viral RNA from transfected cells at early time points did not show any difference between wild type and AP-3deficient cells suggesting that the observed defects at late stages is not due to differences in the transfection efficiencies (Fig. S1). These results clearly demonstrate that the defective viral RNA replication observed in AP-3-deficient cells is not due to disruption of viral entry 
a

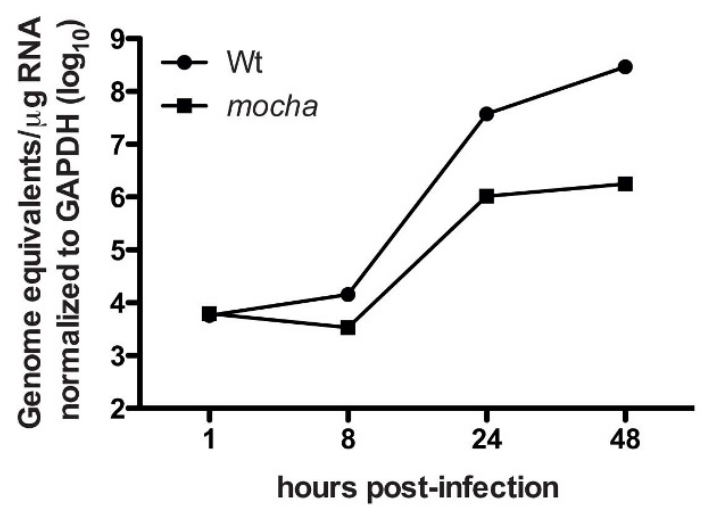

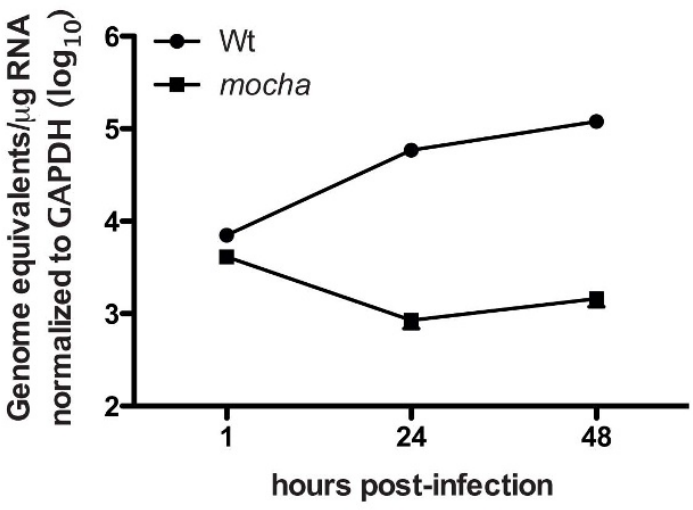

$6 \mathrm{~h}$

$8 \mathrm{~h}$

b

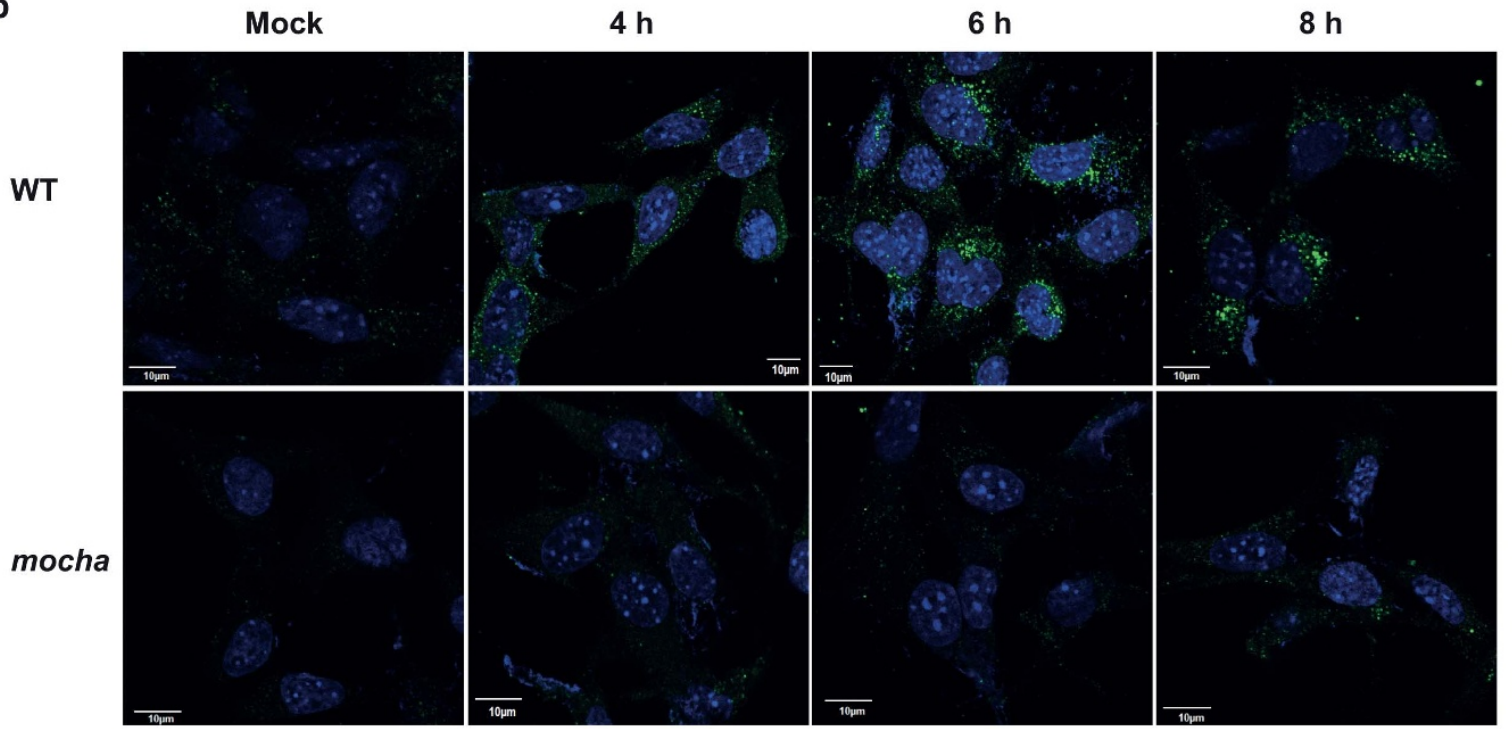

Figure 5 Defective viral RNA replication in AP-3-deficient cells. (a) Cells were infected with JEV (0.3 MOI) or DENV (1 MOI) and at indicated time points infected cells were harvested for RNA isolation and copy number determination by qRT-PCR. Error bars represent Mean \pm SEM. Error bars in some points are not visible on logarithmic scale. Data are representative of two or more experiments performed with three replicates. (b) Cells were grown on coverslips and infected with JEV (MOI of $10 \mathrm{pfu} / \mathrm{cell}$ ). At indicated time points cells were fixed with PFA and stained with anti-dsRNA antibody followed by Alexa- 488 conjugated secondary antibody. The nuclei were stained by DAPI. Scale bar $-10 \mu \mathrm{m}$.

or endosomal fusion processes in these cells. Therefore, it is plausible that some of the cargo proteins sorted by AP-3 at the endosomes are involved in DENV and JEV RNA replication and therefore, these viruses usurp AP-3-mediated trafficking pathway to facilitate viral RNA replication. To test if AP-3 is associated with sites of viral RNA replication, we infected Huh-7 cells with DENV and at $16 \mathrm{~h}$ pi, cells were stained for dsRNA and $\delta$-adaptin. As shown, AP-3 was found to colocalize with most of the dsRNA punctate structures suggesting the recruitment of AP-3 to viral replication compartments (Fig. 7 and Fig. S5). Similar results were obtained with wildtype MEFs infected with JEV where most of the dsRNA colocalized with $\delta$-adaptin (Fig. S3a and S3b). These results suggest that AP-3 deficiency could be affecting viral RNA replication either by a direct involvement in viral RNA replication or by transporting factors essential for viral RNA replication to the compartments where viral replication occurs.

\section{Discussion}

The importance of AP-1 in the biosynthetic and the endo-lysosomal pathways has been demonstrated by animals carrying mutations/ deletions. AP-1 is required for anterograde TGN-to-early endosome as well as retrograde early-endosome-to-TGN transport ${ }^{9-11}$.
Similarly, the role of AP-3 in the biogenesis of lysosomes and lysosome-related organelles such as melanosomes is clearly demonstrated by the phenotype of mice and humans carrying mutations/ deletions in various subunits of the AP-3 complex $^{9,12}$. The mocha mouse (from which the MEFs used in this study was derived) carries a deletion in the Ap3d1 gene which encodes the $\delta 1$ subunit of the AP3 complex. The other three sub-units of the AP- 3 complex are barely detectable in mocha mice suggesting that the $\delta 1$ subunit is essential for maintaining the stability of this heterotetrameric protein complex $^{13}$. In the present study, we show that the heterotetrameric adaptor complexes AP-1 and AP-3 are involved at different stages of infection of RNA viruses belonging to Flaviviridae family. AP-1 was required at later stages of DENV and WNV life-cycle as observed by reduction in the extracellular virus titers and partial colocalization of DENV with AP-1 in the perinuclear area. AP-3 was essential for the initiation of RNA replication of JEV, DENV and WNV. Based on our results we propose a model where AP-3 is involved at a stage post-entry at the stage of viral genome replication (Fig. 8a). AP-1 seems to play a role at later stages of DENV and WNV life-cycle probably post-maturation of the virion or viral egress at the TGN where most of the AP-1 is normally localized (Fig. 8b). This is supported by our data showing increased intracellular DENV 
a

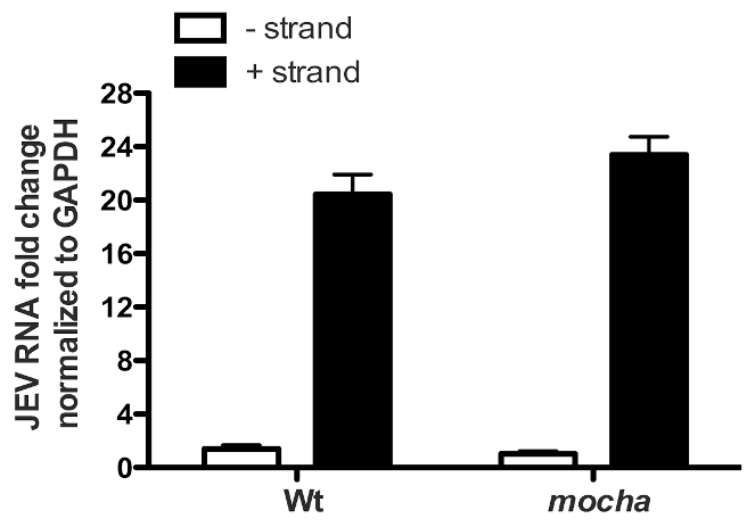

b

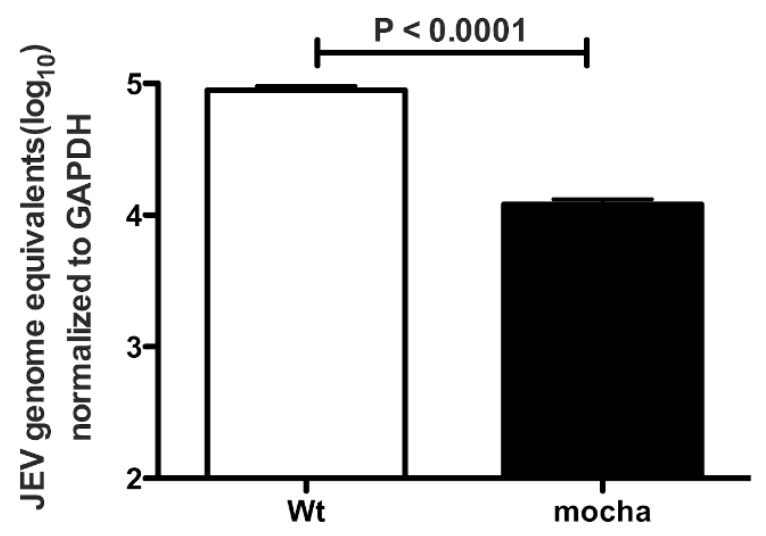

C

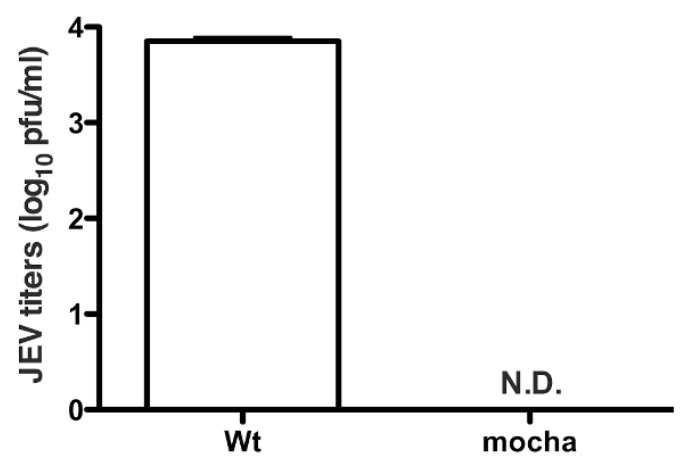

Figure 6 AP-3 is required for viral RNA replication. (a) Cells were infected with JEV ( $3 \mathrm{MOI}$ ) and $24 \mathrm{~h}$ pi cells were harvested for RNA isolation. Positive and negative strand viral RNA was quantitated by qRTPCR as described in methods. GAPDH was used for normalization. Error bars represent Mean \pm SEM. Data are representative of two experiments performed with three replicates. (b-c) Wild type or AP-3-deficient mocha cells were transfected with RNA from JEV-infected cells and $48 \mathrm{~h}$ pi JEV RNA levels were estimated by real time PCR from RNA-transfected cells and viral titers from the supernatant was determined by plaque assay. Error bars represent Mean \pm SEM. N.D. - Not detected.

in AP-1-deficient cells suggesting a role for AP-1 in transport of infectious virus at later stages of viral life-cycle. Our unpublished results and recent reports suggest that JEV infection does not depend on clathrin coats. Our observation that lack of AP-1, which associates with clathrin-coated vesicles, does not affect JEV infection is in line with these observations. Further studies are required to identify if
JEV exits host cells using an alternate pathway which is independent of AP-1 and clathrin-coated vesicles ${ }^{14}$.

AP-3 $\delta$-subunit has been shown to interact with the matrix region of HIV-1 Gag and this interaction was shown to be necessary for Gag trafficking to multi-vesicular bodies and HIV particle formation ${ }^{15-17}$. Understanding the exact functional role of AP-3 in flaviviral lifecycle warrants further investigation. AP-3 may directly be involved in viral replication by binding to one of the viral proteins or indirectly by delivering cargos that are essential for viral RNA replication at the virus-induced replication compartments. We have observed that a number of flaviviral proteins contain the consensus sequence $\mathrm{Yxx} \emptyset$ and $[\mathrm{D} / \mathrm{E}] \mathrm{xxxL}[\mathrm{L} / \mathrm{I}]$ to which $\mu, \beta$ and $\sigma$ sub-units of APs have been shown to bind on other cargo proteins ${ }^{18}$. Thus it remains to be ascertained if any of these viral proteins are capable of binding $\mu 3$ or $\beta$ sub-units. Our study suggests that AP-3-mediated pathways involved in the biogenesis of endosomes/lysosomes play a crucial role in flavivirus RNA replication. Since many earlier reports have shown that flavivirus particles enter the cells reaching early endosomes and subsequently late endosome/lysosome $\mathrm{e}^{19-21}$, it is plausible that AP-3-mediated trafficking in the endo-lysosomal system may be required for initiation of viral replication after release of the viral genome post capsid disassembly. Our infectious RNA transfection data suggests that AP-3 acts at the level of initiation of RNA replication. Some of the potential cargos of AP-3 that may play a role in RNA virus replication could be phosphatidylinositol 4-kinase type II alpha (PI4KIIalpha) and intermediate filament complex ${ }^{22,23}$. Recruitment of AP-1 onto membranes at the TGN has been reported to be dependent on phosphatidylinositol 4-phosphate (PI4P) ${ }^{24}$. Similarly, PI4KIIalpha activity has been shown to be necessary for recuitment of AP-3 onto membranes enriched in PI4P ${ }^{25}$. Some of the earlier studies have identified an important role for other PI4Ks in picornavirus replication and also in heptitis $\mathrm{C}$ virus infection ${ }^{26}$. If PI4KIIalpha plays a similar role in flavivirus life cycle warrants further investigation. Therefore, our future efforts would focus on exploring some of these aspects and characterize the exact role played by AP-3 in viral replication of some of the RNA viruses. In summary, our study has identified AP-1 and AP-3 as crucial components of flavivirus life-cycle and we believe that our observations will help us to further understand the role of APs in the pathogenesis of these globally important pathogens.

\section{Methods}

Cell lines and viruses. All cell lines and their growth conditions have been described before $^{10,27}$. Porcine kidney (PS) cells, Vero and BHK-21 cells were grown in the minimal essential medium (MEM) containing 10\% FBS, Earl's salts and antibiotics. JEV and WNV strain has been described before ${ }^{27}$. DENV-2 (P23085 INDI-60) were obtained from the National Institute of Virology, Pune, India and propagated in $\mathrm{C} 6 / 36$ insect cells in the above medium at $28^{\circ} \mathrm{C}$. Infected culture supernatant was used throughout the study. Viral titers were determined by plaque assay on PS cells for JEV, Vero cells for WNV and BHK-21 cells for DENV as described previously ${ }^{27,28}$.

Virus growth experiments. MEFs were seeded in 24 -well plate $\left(10^{5}\right.$ cells/well $)$ and infected with $0.3 \mathrm{MOI}$ of JEV, 0.3 or $0.1 \mathrm{MOI}$ of WNV and 1 MOI of DENV-2. Virus containing medium (DMEM supplemented with $2 \%$ FBS along with other additives as above) was added to the cells and incubated at $37^{\circ} \mathrm{C}$ on rocker for 1 hour. Virus inoculum was removed and cells were washed twice with PBS and re-fed with normal growth medium. Culture supernatants were collected at the indicated times postinfection and viral titers were determined by plaque assays. After collection of supernatants at above indicated time points, cells were washed once with PBS, trypsinised and resuspended in complete medium. Cells were then centrifuged at $500 \times \mathrm{g}$ for $5 \mathrm{~min}$ at $4^{\circ} \mathrm{C}$. Medium was discarded and cell pellet was resuspended in $250 \mu \mathrm{l}$ Trizol (for isolation of total RNA for quantitative real-time PCR) or in $100 \mu \mathrm{l}$ PBS (for intracellular viral titers). For intracellular viral titers, cells were resuspended in PBS and subjected to three rounds of freeze-thaw cycles by dipping the tubes sequentially in liquid nitrogen and $37^{\circ} \mathrm{C}$ water bath. Cell lysates were centrifuged at $12000 \times \mathrm{g}$ for 10 minutes and intracellular viral titers in the lysed supernatants were determined by plaque assay.

Quantitative real-time PCR. Total cellular RNA was isolated using Trizol reagent. To remove genomic DNA contaminants, $1 \mu \mathrm{g}$ of total RNA was treated with 1.0 unit of RNAse free DNAse I for 30 minutes at $37^{\circ} \mathrm{C}$ and then 10 minutes at $65^{\circ} \mathrm{C}$ to inactivate the DNAse. $100 \mathrm{ng}$ of total RNA was used to determine genome copy 
Mock

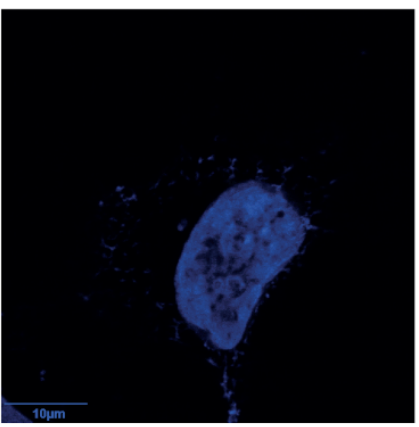

DAPI

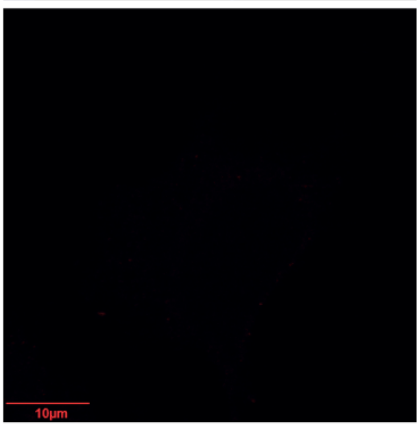

dsRNA
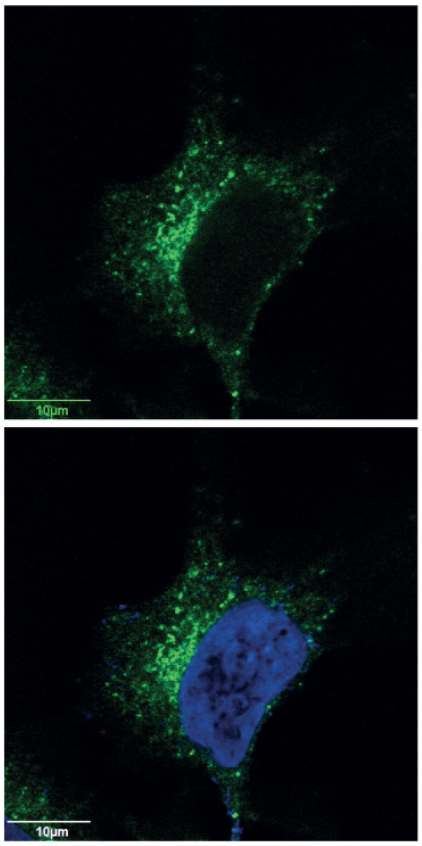

DENV
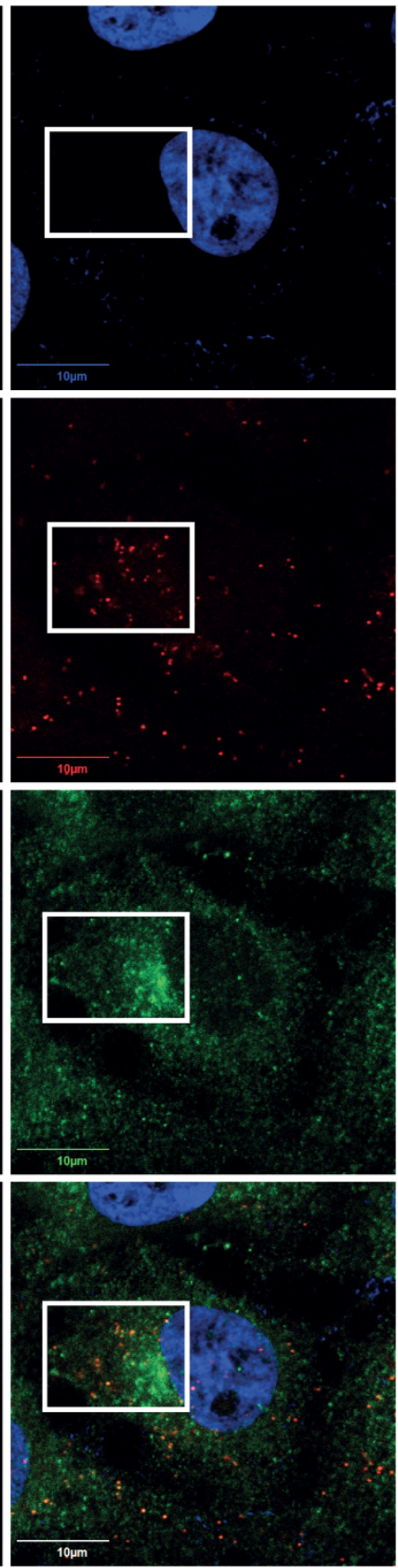

Inset
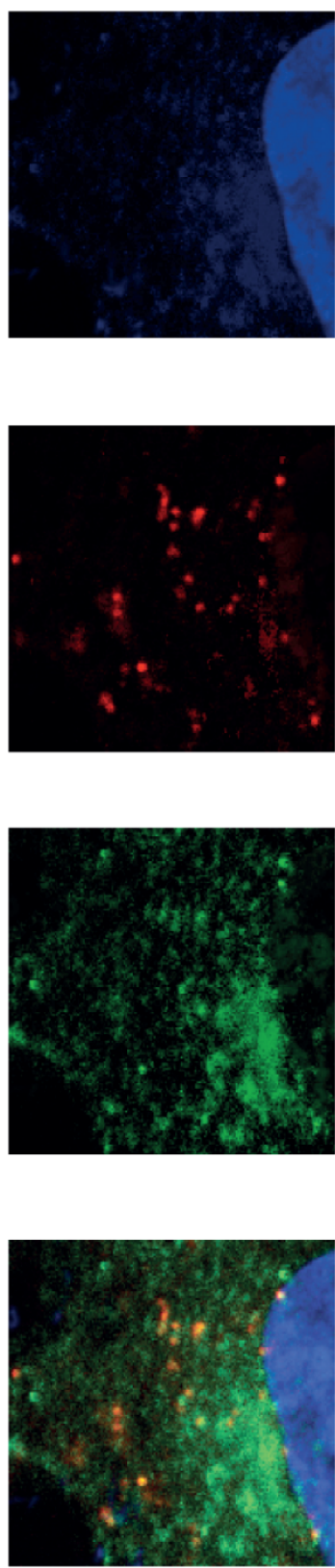

Figure $7 \mid$ AP-3 colocalizes with viral RNA. Huh-7 cells were grown on coverslips and were infected with DENV (MOI of 5 pfu/cell). At 16 h pi cells were fixed and stained with antibodies against dsRNA and $\delta$-adaptin followed by Alexa-546 and Alexa-488-conjugated secondary antibodies. The nuclei were stained by DAPI. Scale bar $-10 \mu \mathrm{m}$. Selected region of colocalization in the overlap image is shown separately in the inset.

numbers using one-step quantitative RT-PCR using primers and probe described previously ${ }^{28-30}$. Each sample was tested in duplicates and viral genome copy numbers in the samples were calculated from a standard curve generated using pre-quantified in vitro transcribed specific RNA standards. Genome copy numbers were normalized to GAPDH mRNA levels (glyceraldehyde-3-phosphate dehydrogenase) [primers: Fwd: 5' -TGTGTCCGTCGTGGATCTGA-3', Rev: 5'-CCTGCTTCACCACCTTC TTGA-3', probe: $5^{\prime}$-FAM-CCGCCTGGAGAAACCTGCCAAGTATG-TAMRA] determined in parallel. Relative expression was calculated using comparative threshold cycle method and viral titers were expressed as genome equivalents/ $\mu \mathrm{g}$ RNA. For detection of positive or negative strand JEV RNA, cDNA synthesis was performed using SuperScript III First-Strand Synthesis System (Invitrogen) with either forward or reverse primers used in qRT-PCR. Fold change in expression of positive and negative strand RNA was quantified by qRT-PCR as desribed above.

Western blot. MEFs were infected with JEV with an MOI of $1 \mathrm{pfu} / \mathrm{cell}$ and at 24 and $48 \mathrm{~h}$ pi, cells were washed with ice-cold PBS and lysed in RIPA buffer (50 mM Tris-Cl $\mathrm{pH}-8.0,150 \mathrm{mM} \mathrm{NaCl}, 1 \%$ IGEPAL CA-630, 0.5\% sodium deoxycholate, $0.1 \%$ SDS,
$1 \times$ protease inhibitor cocktail). Lysates were incubated on ice for $10 \mathrm{~min}$ and centrifuged at $12,000 \times \mathrm{g}$ for $10 \mathrm{~min}$ at $4^{\circ} \mathrm{C}$. Supernatants were collected and boiled in $4 \times$ Laemmli buffer and resolved on SDS-PAGE and transferred onto membranes, incubated with primary antibodies (mouse anti- $\beta$-actin (Sigma-Aldrich), rabbit polyclonal anti-JEV capsid raised in-house) at $4^{\circ} \mathrm{C}$ overnight followed by three washes with TBST and one hour incubation with HRP-conjugated secondary antibodies. Blots were washed three times with TBST and once with TBS and developed by enhanced chemiluminescence substrate.

Immunofluorescence. Cells grown on coverslips were infected with JEV or DENV with indicated MOIs. At indicated time points, cells were washed twice in PBS containing $1.0 \mathrm{mM} \mathrm{Ca}^{2+}$ and $0.5 \mathrm{mM} \mathrm{Mg}^{2+}$ and then fixed in $3 \%$ paraformaldehyde for $20 \mathrm{~min}$. Samples were quenched with PBS containing $0.1 \mathrm{M}$ glycine and then washed twice with PBS. Cell membranes were permeabilized with PBS containing $0.1 \%$ Triton X-100 for 5 min and then non-specific antibody binding sites were blocked by incubating with PBS containing $2 \%$ normal goat serum for $10 \mathrm{~min}$ at room temperature (RT). Fixed cells were washed three times with PBS-Triton buffer, 
a
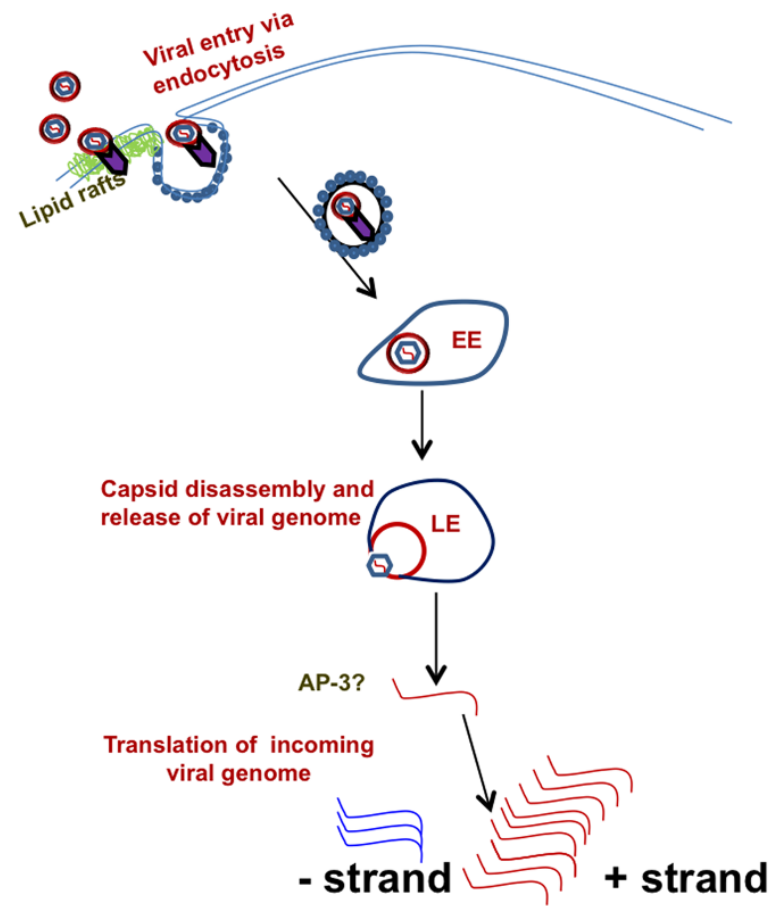

AP-3? Viral genome replication b

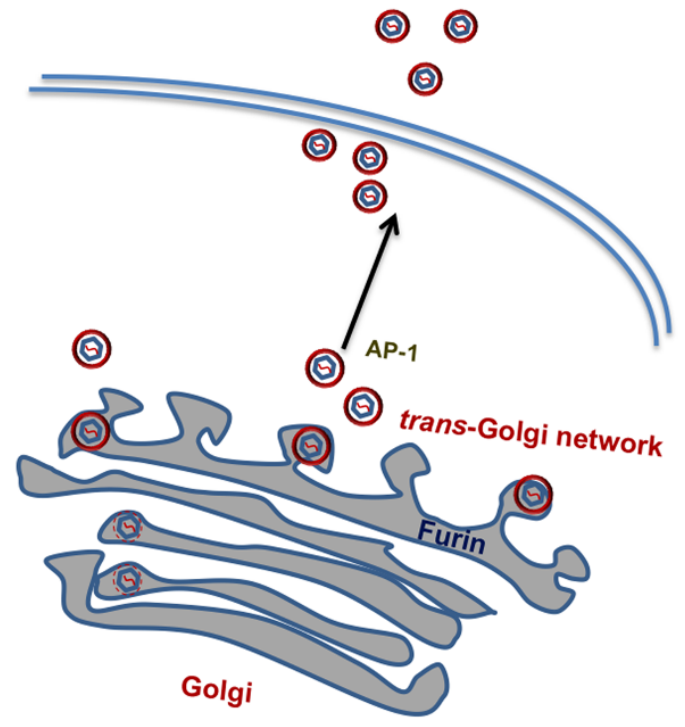

Figure 8 Role of AP-1 and AP-3 in flavivirus life-cycle. (a) Flaviviruses bind to attachment factors/receptors at the plasma membranes or in lipid rafts and are internalized. Virus-receptor complexes are delivered to early endosomes, which may mature into late endosomes. Fusion of viral and host membranes may occur in early or late endosomes leading to release of naked capsids which disassemble to release viral RNA into the cytoplasm for translation followed by viral RNA replication via negative strand intermediates. The possible step at which AP-3 may be involved is indicated.

(b) Immature virions traverse through the Golgi stack and enter the TGN where furin-mediated cleavage between prM-M renders the virion infectious due to conformational changes. Infectious virions exit the TGN and are secreted out of the cells presumably by the constitutive secretory pathway. AP-1 is possibly involved at the egress stage from the TGN.

followed by incubation with one or more of the following antibodies: anti-TGN46 (AbD Serotec), anti dsRNA antibody J2 (English \& Scientific Consulting Bt., Hungary), anti-delta adaptin antibody (SA-4) and LAMP-1 antibody (H4A3) (Developmental Studies Hybridoma Bank from the University of Iowa) or mouse anti-human delta adaptin antibody (BD Biosciences), anti-gamma adaptin antibody (BD Biosciences) and anti-DENV-capsid polyclonal antibody generated in-house against a purified recombinant dengue capsid protein. Cells were incubated with primary antibodies for $1 \mathrm{~h}$ at RT, washed three times with PBS-Triton buffer and incubated with secondary antibodies conjugated with Alexa-flour dyes for $1 \mathrm{~h}$ at RT. Coverslips were mounted onto microscope slides using ProLong Gold Antifade containing DAPI and left overnight at RT. Images were acquired using $20 \times$ or $100 \times$ objective in Olympus FLUOVIEW FV1000 confocal microscope.

Viral RNA transfection. Wild type MEFs were infected with JEV at an MOI of $0.3 \mathrm{pfu} / \mathrm{cell}$ and $48 \mathrm{~h}$ pi RNA was isolated from infected cells and used for transfecting wild type and AP-3 deficient MEFs in a 24-well plate $\left(7.5 \times 10^{4}\right.$ cells/well $)$ using Lipofectamine 2000. Transfection media was replaced with complete media with antibiotics after about 14 hours post-transfection. Supernatants were collected at $48 \mathrm{~h}$ post-transfection for plaque assay and cells were collected in Trizol for detection of viral RNA by qRT-PCR using one-step PCR Taqman reagents. For determination of transfection efficiency, the amount of input RNA was measured at $8 \mathrm{~h}$ posttransfection by preparing total RNA and estimating the copy numbers by qRT-PCR as described above.

Statistical analysis. GraphPad Prism software was used for all graphical representations and statistical analysis. All experiments were performed in two or three replicates samples and each figure is representative of experiments performed at least three times. P values were estimated by non-parametric, unpaired, two-tailed t-test.

1. Lindenbach, B. D., Thiel, H. J. \& Rice, C. M. Flaviviridae: The Viruses and Their Replication. Fields Virology I, 1101-1152 (2007).

2. Smit, J., Moesker, B., Rodenhuis-Zybert, I. \& Wilschut, J. Flavivirus Cell Entry and Membrane Fusion. Viruses 3, 160-171 (2011).
3. Medigeshi, G. R. Mosquito-borne flaviviruses: overview of viral life-cycle and host-virus interactions. Future Virology 6, 1075-1089 (2011).

4. Braulke, T. \& Bonifacino, J. S. Sorting of lysosomal proteins. Biochimica et biophysica acta 1793, 605-614 (2009).

5. Luzio, J. P., Gray, S. R. \& Bright, N. A. Endosome-lysosome fusion. Biochem Soc Trans 38, 1413-1416 (2010).

6. Hirst, J. et al. The Fifth Adaptor Protein Complex. PLoS Biol 9, e1001170 (2011).

7. Krishnan, M. N. et al. RNA interference screen for human genes associated with West Nile virus infection. Nature 455, 242-245 (2008).

8. Wang, P.-G. et al. Efficient Assembly and Secretion of Recombinant Subviral Particles of the Four Dengue Serotypes Using Native prM and E Proteins. PLoS ONE 4, e8325 (2009).

9. Meyer, C. et al. mu1A-adaptin-deficient mice: lethality, loss of AP-1 binding and rerouting of mannose 6-phosphate receptors. Embo J 19, 2193-2203 (2000).

10. Medigeshi, G. R. \& Schu, P. Characterization of the in vitro retrograde transport of MPR46. Traffic 4, 802-811 (2003).

11. Reusch, U., Bernhard, O., Koszinowski, U. \& Schu, P. AP-1A and AP-3A lysosomal sorting functions. Traffic 3, 752-761 (2002).

12. Dell'Angelica, E. C. AP-3-dependent trafficking and disease: the first decade. Current Opinion in Cell Biology 21, 552-559 (2009).

13. Kantheti, P. et al. Mutation in AP-3 delta in the mocha mouse links endosomal transport to storage deficiency in platelets, melanosomes, and synaptic vesicles. Neuron 21, 111-122 (1998).

14. Zhu, Y. Z. et al. Japanese encephalitis virus enters rat neuroblastoma cells via a pHdependent, dynamin and caveola-mediated endocytosis pathway. J Virol 86, 13407-13422 (2012).

15. Dong, X. et al. AP-3 directs the intracellular trafficking of HIV-1 Gag and plays a key role in particle assembly. Cell 120, 663-674 (2005).

16. Garcia, E., Nikolic, D. S. \& Piguet, V. HIV-1 replication in dendritic cells occurs through a tetraspanin-containing compartment enriched in AP-3. Traffic 9 , 200-214 (2008)

17. Liu, L. et al. Defective HIV-1 Particle Assembly in AP-3-Deficient Cells Derived from Patients with Hermansky-Pudlak Syndrome Type 2. Journal of virology 86, 11242-11253 (2012).

18. Bonifacino, J. S. \& Traub, L. M. Signals for sorting of transmembrane proteins to endosomes and lysosomes. Annu Rev Biochem 72, 395-447(2003). 
19. Chu, J. J. H. \& Ng, M. L. Infectious Entry of West Nile Virus Occurs through a Clathrin-Mediated Endocytic Pathway. J. Virol. 78, 10543-10555 (2004).

20. Krishnan, M. N. et al. Rab 5 Is Required for the Cellular Entry of Dengue and West Nile Viruses. J. Virol. 81, 4881-4885 (2007).

21. van der Schaar, H. M. et al. Dissecting the cell entry pathway of dengue virus by single-particle tracking in living cells. PLoS Pathog 4, e1000244 (2008).

22. Craige, B., Salazar, G. \& Faundez, V. Phosphatidylinositol-4-kinase type II alpha contains an AP-3-sorting motif and a kinase domain that are both required for endosome traffic. Molecular Biology of the Cell 19, 1415-1426 (2008).

23. Styers, M. L. et al. The Endo-Lysosomal Sorting Machinery Interacts with the Intermediate Filament Cytoskeleton. Molecular Biology of the Cell 15, 5369-5382 (2004).

24. Wang, Y. J. et al. Phosphatidylinositol 4 phosphate regulates targeting of clathrin adaptor AP-1 complexes to the Golgi. Cell 114, 299-310 (2003).

25. Salazar, G. et al. Hermansky-Pudlak syndrome protein complexes associate with phosphatidylinositol 4-kinase type II alpha in neuronal and non-neuronal cells. J Biol Chem 284, 1790-1802 (2009).

26. Delang, L., Paeshuyse, J. \& Neyts, J. The role of phosphatidylinositol 4-kinases and phosphatidylinositol 4-phosphate during viral replication. Biochemical pharmacology 84, 1400-1408 (2012).

27. Haridas, V. et al. Bispidine-Amino Acid Conjugates Act as a Novel Scaffold for the Design of Antivirals That Block Japanese Encephalitis Virus Replication. PLoS Negl Trop Dis 7, e2005 (2013).

28. Hirsch, A. J. et al. The Src family kinase c-Yes is required for maturation of West Nile virus particles. J Virol 79, 11943-11951 (2005).

29. Gurukumar, K. R. et al. Development of real time PCR for detection and quantitation of Dengue Viruses. Virology Journal 6, 10 (2009).
30. Huang, J. L. et al. Sensitive and specific detection of strains of Japanese encephalitis virus using a one-step TaqMan RT-PCR technique. J Med Virol 74, 589-596 (2004).

\section{Acknowledgments}

We thank Rajgokul K.S. for technical assistance. T.A. is supported by vaccine research innovation award of the institute. This study was supported by the institutional grant by the Department of Biotechnology to G.M.

\section{Author contributions}

T.A. performed all the experiments. G.M. designed and performed the experiments, analysed the data and wrote the manuscript. P.S. contributed reagents and analysed the data. All authors reviewed the manuscript.

\section{Additional information}

Supplementary information accompanies this paper at http://www.nature.com/ scientificreports

Competing financial interests: The authors declare no competing financial interests.

License: This work is licensed under a Creative Commons

Attribution-NonCommercial-NoDerivs 3.0 Unported License. To view a copy of this license, visit http://creativecommons.org/licenses/by-nc-nd/3.0/

How to cite this article: Agrawal, T., Schu, P. \& Medigeshi, G.R. Adaptor protein complexes- 1 and 3 are involved at distinct stages of flavivirus life-cycle. Sci. Rep. 3, 1813; DOI:10.1038/srep01813 (2013) 
SUBJECT AREAS:

VIRUS-HOST INTERACTIONS

DENGUE VIRUS MECHANISMS OF DISEASE COAT COMPLEXES

\section{SCIENTIFIC REPORTS:}

$3: 1813$

DOI: $10.1038 /$ srep0 01813

(2013)

Published:

9 May 2013

Updated:

13 June 2014
CORRIGENDUM: Adaptor protein complexes-1 and 3 are involved at distinct stages of flavivirus life-cycle

Tanvi Agrawal, Peter Schu \& Guruprasad R. Medigeshi

The Acknowledgements section in this Article is incomplete; it should include the following statement: "P.S. is funded by the grant DFG Schu 802/3-2”. 